\title{
A NEW PROOF OF THE PARACOMPACTNESS OF METRIC SPACES
}

\section{DONALD ORNSTEIN}

The purpose of this paper is to give a new proof of A. H. Stone's Theorem that every metric space is paracompact. ${ }^{1}$ We feel that the combinatorics involved are of some interest in their own right and that our method may be useful in other contexts. The statement that a space $X$ is paracompact means that every open cover of $X$ has a locally finite open refinement that covers $X$. (An open refinement of a collection of open sets is a new collection of open sets such that each set in the new collection is contained in some set of the original collection. A cover is said to be locally finite if each point has a neighborhood intersecting only a finite number of elements in the cover.)

Proof. (A) We shall first get a refinement such that each point is covered only a finite number of times.

To do this we will order the elements of the given cover. Call the cover $\left\{C_{\alpha}\right\}$. Let $R(x, m)$ be the open circle of radius $1 / 2^{m}$ about $x$. A chosen circle (with respect to $C_{\alpha}$ ) is a circle $R\left(x, n_{x}+1\right)$ such that (i) $R\left(x, n_{x}\right) \subset C_{\alpha}$, (ii) $n_{x}$ is the smallest integer for which (i) holds and (iii) $R\left(x, n_{x}\right) \subset C_{\beta}$ for some $\beta<\alpha$. Let $\chi_{\alpha}$ be $C_{\alpha}$ minus the closure of the union of all the chosen circles [by $C$ minus $B, \mathrm{I}$ mean $C$ intersect the complement of $B] .\left\{C_{\alpha}\right\}$ is the refinement we want for (A).

We will show that $\left\{\mathcal{C}_{\alpha}\right\}$ is a cover. Assume we did not cover some point $x$. Let $C_{\alpha}$ be the first element of the original cover to contain $x$. $x \notin \tilde{C}_{\alpha}$ and, since $x$ is not in a chosen circle, $x$ must be a limit point of chosen cirrles (with respect to $C_{\mathrm{e}}$ ). [Define the expansion of $R(x, m)$ to be $R(x, m-1)$.] One of the expansions of the chosen circles must contain $x$, for if we approach $x$ with a sequence of chosen circles their radius can not approach 0 . But if $x$ is in the expansion of some chosen circle it must be contained in a previous $C_{\beta}$.

We now show that each point is contained in only a finite number of $\bar{C}_{\alpha}$. If $\bar{C}_{\beta}$ is to contain $x$ then $C_{\beta}$ must be the first element in $\left\{C_{\alpha}\right\}$ to contain some $R(x, m)$. If $C_{\beta}$ is the first to contain $R(x, m), C_{\gamma}$ the first to contain $R(x, n)$ and if $n>m$ then $\gamma \leqq \beta$. Hence the $\widetilde{C}_{\alpha}$ that contain $x$ form a descending sequence of ordinals and therefore only a finite number are distinct.

Received by the editors January 29, 1968.

${ }^{1}$ Mary Ellen Rudin has yet another proof of this theorem which is different but just as simple as ours. 
(B) We shall now get a locally finite refinement of $\left\{\tilde{C}_{\alpha}\right\}$. For each $x$ let $m_{x}$ be $\frac{1}{2}$ the sup of the radius of circles about $x$ that are contained in some $\tilde{C}_{\alpha}$ [we can assume that each $m_{x}$ is finite because otherwise our problem has a trivial solution]. Let $S(x, n)$ be the open circle of radius $n$ about $x$. Let $\widetilde{C}_{\alpha}^{\prime}$ be the union of all $S\left(x, m_{x} / 2\right)$ such that $\tilde{C}_{\alpha}$ is the first of the $\left\{\tilde{C}_{\alpha}\right\}$ to contain $S\left(x, m_{x}\right) .\left\{\tilde{C}_{\alpha}^{\prime}\right\}$ is a locally finite cover and $\tilde{C}_{\alpha}^{\prime} \subset C_{\alpha}$.

To show local finiteness, it is enough to show that if $\widetilde{C}_{\alpha}^{\prime} \cap S\left(x, m_{x} / 8\right)$ $\neq 00$ then $x \in \tilde{C}_{\alpha}$. Let us assume the contrary, i.e. for some $y, S\left(y, m_{y} / 2\right)$ $\cap S\left(x, m_{x} / 8\right) \neq 0$ and that $x \notin S\left(y, m_{y}\right)$. Then $m_{y} / 2<m_{x} / 8$ and $y \in S\left(x, m_{x} / 4\right)$. But then $S\left(y, 5 / 2 m_{y}\right) \subset S\left(x, m_{x}\right)$ and hence is in some $\tilde{C}_{\alpha}$ contradicting the maximum property of $S\left(y, m_{y}\right)$.

STANFORd UNIVERSITY 